Artículos

\title{
Validación de constructo de la escala Zung en pacientes con falla cardíaca*
}

\section{Zung Scale Construct Validation in Heart Failure Patients}

Validação do construto da escala de Zung em pacientes com insuficiência cardíaca

Lyda Z. Rojas ${ }^{\text {a }}$

DOI: https://doi.org/10.11144/Javeriana.ie23.vcez

Fundación Cardiovascular, Colombia

lydarojas@fcv.org

ORCID: https://orcid.org/0000-0002-4252-7105

Recibido: 28 Septiembre 2020

Aceptado: 10 Junio 2021

Silvia Juliana Trujillo-Cáceres

Fondo Colombiano de Enfermedades de Alto Costo,

Colombia

ORCID: https://orcid.org/0000-0002-7626-3822

Juliana Alexandra Hernández-Vargas

Fondo Colombiano de Enfermedades de Alto Costo,

Colombia

ORCID: https://orcid.org/0000-0001-6183-252X

Adriana Milena Jurado

Fundación Cardiovascular de Colombia, Colombia

ORCID: https://orcid.org/0000-0002-3137-8319

Luis E. Echeverría

Fundación Cardiovascular de Colombia, Colombia

ORCID: https://orcid.org/0000-0001-5784-3859

Sergio Alejandro Gómez-Ochoa

Fundación Cardiovascular de Colombia, Colombia

ORCID: https://orcid.org/0000-0002-1396-5042

Zayne Milena Roa-Diaz

University of Bern, Suiza

ORCID: https://orcid.org/0000-0002-0847-0969

\section{Resumen:}

Introducción: la escala Zung para depresión ha sido previamente utilizada en pacientes con falla cardíaca; sin embargo, en nuestro conocimiento no se encuentra evidencia de su validez para la versión en español, ni su uso en población adulta con falla cardíaca en Colombia. Objetivo: determinar la validez de constructo de la escala Zung para depresión en su versión original y proponer una versión abreviada para pacientes adultos con falla cardíaca. Método: estudio de corte transversal, realizado en 200 pacientes de una clínica de falla y trasplante cardiaco, a quienes se aplicó la Zung Self-Rating Depression Scale. Se evaluó la consistencia interna por medio del alfa de Cronbach, y el análisis factorial fue utilizado para identificar las dimensiones del instrumento. Resultados: la consistencia interna de la versión original de la escala (20 ítems) fue de $\alpha=0,811$. El análisis de factores mostró una estructura compuesta por tres de ellos que explican el 51,59\% de la varianza total. La nueva versión abreviada (13 ítems) obtuvo un $\alpha=$ 0,819, y los ítems correlacionaron con un único factor que explicó el 33,54\% de la varianza total. Discusión: Nuestros hallazgos son similares a los encontrados por otros autores en diferentes poblaciones a la estudiada. Conclusiones: se evidenció validez de constructo tanto para la escala Zung, para depresión, en su versión original, como para la abreviada, creada en la población estudiada. Sin embargo, se requieren estudios adicionales que verifiquen estos hallazgos en una muestra representativa, y que otros aspectos de la psicometría sean evaluados.

Notas de autor

${ }^{a}$ Autora de correspondencia. Correo electrónico: lydarojas@fcv.org 
Palabras clave: estudio de validación, escalas de valoración psiquiátrica, depresión, insuficiencia cardíaca.

\section{Abstract:}

Introduction: the Zung scale for depression has previously been used in patients with heart failure; However, to our knowledge, there is no evidence of its validity for the Spanish version, nor its use in the adult population with heart failure in Colombia. Objective: to determine the construct validity of the Zung scale for depression in its original version and propose an abbreviated version for adult patients with heart failure. Method: A cross-sectional study was carried out in 200 patients from a heart failure and transplant clinic to whom the Zung Self-Rating Depression Scale was applied. Internal consistency was evaluated using Cronbach's alpha, and factor analysis was used to identify the instrument's dimensions. Results: the internal consistency of the original version of the scale (20 items) was $\alpha=0.811$. The factor analysis showed a structure composed of three that explains $51.59 \%$ of the total variance. The new abbreviated version (13 items) obtained $\alpha=0.819$, and the items correlated with a single factor that explained $33.54 \%$ of the total variance. Discussion: Our findings are similar to those found by other authors in different populations from the one studied. Conclusions: construct validity was evidenced for both the Zung scale for depression, in its original version, and the abbreviated version created in the studied population.

Keywords: validation study, psychiatric rating scales, depression, heart failure.

\section{Resumo:}

Introdução: a escala de Zung para depressão foi usada anteriormente em pacientes com insuficiência cardíaca. No entanto, até onde sabemos, não há evidências de sua validade para a versão em espanhol, nem seu uso na população adulta com insuficiência cardíaca na Colômbia. Objetivo: determinar a validade de construto da escala de Zung para depressão em sua versão original e propor uma versão abreviada para pacientes adultos com insuficiência cardíaca. Método: estudo transversal, realizado com 200 pacientes de uma clínica de insuficiência cardíaca e transplante, aos quais foi aplicada a Zung Self-Rating Depression Scale. A consistência interna foi avaliada por meio do alfa de Cronbach e a análise fatorial foi utilizada para identificar as dimensões do instrumento. Resultados: a consistência interna da versão original da escala (20 itens) foi $\alpha=0,811$. A análise fatorial mostrou uma estrutura composta por três deles que explicam $51,59 \%$ da variância total. A nova versão abreviada (13 itens) obteve $\alpha=0,819$, e os itens se correlacionaram com um único fator que explicou 33,54\% da variância total. Discussão: Nossos achados são semelhantes aos encontrados por outros autores em populações diferentes da estudada. Conclusões: a validade de construto foi evidenciada tanto para a escala de Zung, para depressão, em sua versão original, quanto para a versão abreviada, elaborada na população estudada. No entanto, estudos adicionais são necessários para verificar esses achados em uma amostra representativa e que forem avaliados outros aspectos da psicometria.

Palavras-chave: estudo de validação, escalas de valoração psiquiátrica, depressão, insuficiência cardíaca.

\section{Introducción}

La depresión es una de las principales causas de discapacidad y mortalidad prematura en el mundo, y afecta aproximadamente a 350 millones de personas (1). Se ha descrito que los pacientes con enfermedades cardiovasculares tienen una mayor prevalencia de problemas de salud mental (2). La depresión es una comorbilidad común en los pacientes con falla cardíaca (FC) y está asociada con peores desenlaces clínicos, sin embargo, es poco reconocida $(3,4)$. Se ha determinado una prevalencia de depresión del $21,5 \%$ en pacientes con FC, y esta puede variar de acuerdo al uso de cuestionarios o entrevista diagnóstica (33,6 y 19,3 $\%$, respectivamente) y la severidad de la FC ( $11 \%$ clase I vs. $42 \%$ clase NYHA IV), entre otros factores (5). Además, se relaciona con mayores síntomas somáticos, hospitalizaciones, carga financiera, peor calidad de vida y autocuidado, mayor uso de los servicios de salud y morbi-mortalidad (6-8).

La depresión ha demostrado ser un predictor de eventos cardiovasculares, independientemente de la gravedad de la FC, por lo que hace necesario considerar su evaluación como otro factor de riesgo importante en esta población (6,7). La depresión puede tener consecuencias iguales, o incluso más negativas, en comparación con los factores de riesgo tradicionales como la diabetes, hipertensión, tabaquismo e hipercolesterolemia (9). Asimismo, las guías de manejo para la FC recomiendan, en el plan de cuidados, mantener la vigilancia de aspectos psicosociales, conductuales y socioeconómicos de los pacientes con FC y sus cuidadores. Lo anterior incluye el acceso a los cuidados y las desigualdades en la atención médica; dentro 
de los aspectos psicosociales se destacan temas específicos del género, la actividad sexual y el tamizaje para la depresión (8).

También es de resaltar que el estudio OPTIMIZE-HF demostró que los pacientes depresivos son un grupo vulnerable entre los pacientes con FC, así que es de suma importancia un diagnóstico preciso y oportuno en esta población (10). Adicionalmente, a medida que los pacientes con FC envejecen, la supervivencia, como el principal resultado de la atención, es reemplazada por medidas relevantes de las funciones social, emocional, física y mental (11).

Escalas para la depresión como Beck Depression Inventory (BDI), Center for Epidemiological Studies Depression Scale (CES-D), Hamilton Rating Scale for Depression (HRSD) y Zung Self-Rating Depression Scale (SDS) son ampliamente utilizadas como herramientas de tamizaje tanto para el apoyo diagnóstico como para investigación $(12,13)$. El SDS, denominada en español como Escala Zung para depresión (EZ-D), ha sido previamente utilizada en pacientes con falla cardíaca (6, 14-16); sin embargo, en nuestro conocimiento no se encuentra evidencia de su validez para la versión en español en población adulta colombiana con FC. En Colombia, principalmente se han realizado estudios sobre su validez en adolescentes $(17,18)$, adultos jóvenes (universitarios) $(19,20)$ y adultos de la población general de Bucaramanga $(21,22)$.

Por lo anterior, el objetivo del estudio fue determinar la validez de constructo de la EZ-D en su versión original, y proponer una versión abreviada en pacientes adultos con FC.

\section{Método}

Se realizó un estudio de corte transversal en una institución de cuarto nivel de complejidad en Colombia, durante el 2015. Se incluyeron personas mayores de 18 años con diagnóstico confirmado de FC y se excluyó a quienes presentaron alteraciones de su esfera mental o limitaciones en la comunicación, que impidieran el diligenciamiento de la EZ-D.

El tamaño de la muestra fue calculado teniendo en cuenta la recomendación de 10 personas por ítem analizado, considerado un tamaño adecuado para la ejecución de un análisis factorial (20), para un total de 200 participantes. La muestra fue seleccionada de forma no probabilística; todos los pacientes fueron invitados a participar consecutivamente por una enfermera previamente capacitada que realizó las entrevistas en las citas de control médico.

El equipo investigador consolidó un formato de recolección de datos que incluyó características sociodemográficas, clínicas y los ítems del EZ-D. La EZ-D para la depresión es un instrumento de autodiligenciamiento, que consta de 20 ítems calificados en una escala tipo Likert que va desde 1 (nunca o muy pocas veces) a 4 (la mayoría del tiempo o siempre), $50 \%$ en sentido positivo y $50 \%$ en sentido inverso. El puntaje total crudo oscila entre 20 y 80, con un punto de corte de 40 para los síntomas depresivos de importancia clínica $(20,23)$. La escala incluye la evaluación de síntomas comunes en el diagnóstico de la depresión, agrupados en los siguientes componentes: I) alteraciones del estado de ánimo, caracterizadas por sentimientos profundos y quejas de estar deprimido, triste, abatido y lloroso; II) síntomas fisiológicos como la variación diurna, alteraciones del sueño, disminución del apetito, peso, libido, presencia de estreñimiento, taquicardia y fatiga inexplicable; III) distorsiones psicomotoras (quietud o agitación); y IV) alteraciones psicológicas que incluyen la confusión, vacío, desesperanza, indecisión, irritabilidad, insatisfacción, devaluación personal e ideación suicida $(17,24,25)$. Esta escala ha demostrado propiedades psicométricas aceptables $(12,13,26)$, y se encuentra entre las más utilizadas para la evaluación de la depresión en las personas con FC, tanto en contextos clínicos como de investigación, especialmente en ensayos clínicos para determinar el efecto de intervenciones farmacológicas y no farmacológicas en personas en situación de depresión $(1,12,27)$. 
La validez de constructo de la EZ-D se evaluó con la consistencia interna por medio del coeficiente alfa de Cronbach (28) y el análisis factorial exploratorio para identificar los factores principales del instrumento. Se utilizó esta aproximación debido a que, aunque previamente se ha descrito su estructura factorial, esta puede cambiar en función de la muestra y las características particulares de la población con FC (29). Se inició el análisis factorial con la estimación del estadístico Kayser-Meyer-Olkin (KMO) y la prueba de esfericidad de Bartlett para establecer la pertinencia del análisis factorial; considerando como adecuados valores de KMO $\geq 0,7 \mathrm{y}$ un alto valor de $\chi 2$, acompañado de un valor de $\mathrm{p}<0,05$, respectivamente $(30,31)$. Teniendo en cuenta que la escala de medición de la EZ-D es tipo Likert, sería apropiado utilizar la matriz de correlaciones policóricas. Sin embargo, se han descrito algunos supuestos fundamentales para su utilización, relacionados principalmente con la adecuación del tamaño muestral. En este sentido, no es recomendable emplear este método en muestras inferiores a 300 sujetos, tal es el caso de nuestro análisis, debido a que se puede afectar el nivel de precisión y estabilidad de las estimaciones (32). Por lo anterior, la extracción de factores se realizó con el método de análisis de componentes principales (ACP), teniendo como criterio autovalores superiores a 1,4 y coeficientes de correlación de los factores con valor $\geq 0,30$, con el propósito de evitar la sobreestimación de factores considerados importantes $(17,21,33)$. Se realizó una rotación oblicua (Promax) teniendo en cuenta una alta relación entre los ítems (33). Además, para explorar el nivel de dificultad de los ítems del instrumento en la población del estudio, se elaboró el mapa de Wright ítem-persona (34).

Para la construcción de una versión abreviada de la EZ-D, se tuvieron en cuenta los criterios previamente descritos por otros autores $(20,21)$ : se seleccionaron ítems que tuvieran una correlación de Pearson o Spearman $\geq 0,50$ con respecto al puntaje total de la escala. De la misma forma descrita anteriormente, la validez de constructo de esta versión fue evaluada. Los análisis se realizaron en el programa estadístico Stata v15 y Winsteps V3.69.1 (Chicago, Illinois, USA).

En relación con los aspectos éticos, se siguieron los lineamientos de las normas científicas, técnicas y administrativas para la investigación en salud, presentados en la Resolución 008430 de 1993 del Ministerio de Salud de Colombia y de la Declaración de Helsinki. Asimismo, este estudio fue catalogado como una investigación sin riesgo para los sujetos humanos. Cada paciente recibió un código no vinculante para garantizar su anonimato durante todas las etapas de la investigación. Se contó con el aval del Comité de Ética de la institución, y todos los participantes dieron su consentimiento informado por escrito.

\section{Resultados}

Las características sociodemográficas y clínicas de la muestra se han descrito previamente en otra publicación (35).

La consistencia interna de la escala EZ-D, versión original constituida por 20, ítems fue $\alpha=0,811$ (IC 95 $\% 0,779$ a 0,843). La prueba de esfericidad de Bartlett fue $\chi^{2}(190)=1009,53, p=0.000$ La prueba de adecuación de la muestra KMO obtuvo un valor general de 0,802. El análisis de factores mostró una estructura compuesta por tres de ellos que explican el 51,59\% de la varianza total. El primer componente representó los aspectos afectivos y físicos (19,81\%); el segundo, los síntomas somáticos (16,84\%); y el tercero, los síntomas cognitivos (14,94\%) (tabla 1). El mapa de Wright identificó al ítem 19 "siento que los demás estarían mejor si yo muriera” como el más difícil de responder, mientras que los más fáciles fueron los ítems 12 y 2 "me resulta fácil hacer las cosas que acostumbraba hacer" y "en la mañana es cuando me siento mejor", respectivamente (figura 1). 
TABLA 1.

Análisis factorial de componentes principales con rotación oblicua de la escala de Zung para depresión (original) en pacientes con falla cardíaca $(\mathrm{n}=200)$

\begin{tabular}{|c|c|c|c|c|c|c|}
\hline \multirow{2}{*}{\multicolumn{2}{|c|}{ Ítems }} & \multicolumn{3}{|c|}{ Componente } & \multirow[b]{2}{*}{ Singularidad } & \multirow[b]{2}{*}{ Comunalidad ${ }^{*}$} \\
\hline & & 1 & 2 & 3 & & \\
\hline 3 & $\begin{array}{l}\text { Tengo periodos de llanto o } \\
\text { deseos de llorar }\end{array}$ & 0,7014 & & & 0,4996 & 0,5004 \\
\hline 13 & $\begin{array}{l}\text { Me siento intranquilo y no } \\
\text { puedo mantenerme quieto }\end{array}$ & 0,6833 & & & 0,5061 & 0,4939 \\
\hline 9 & $\begin{array}{l}\text { E1 corazón me late más rápido } \\
\text { que de costumbre }\end{array}$ & 0,6778 & & & 0,6130 & 0,3870 \\
\hline 15 & Estoy más irritable de lo usual & 0,6488 & & & 0,5844 & 0,4156 \\
\hline 1 & Me siento decaido y triste & 0,6082 & & & 0,4951 & 0,5049 \\
\hline 10 & $\begin{array}{l}\text { Me canso, aunque no haga } \\
\text { nada }\end{array}$ & 0,4730 & & & 0,5444 & 0,4556 \\
\hline 4 & $\begin{array}{l}\text { Me cuesta trabajo dormirme en } \\
\text { la noche }\end{array}$ & 0,3751 & & & 0,7132 & 0,2868 \\
\hline 8 & $\begin{array}{l}\text { Tengo molestias de } \\
\text { estreñimiento }\end{array}$ & 0,3209 & & & 0,7939 & 0,2061 \\
\hline 17 & Siento que soy útil y necesario & & 0,8018 & & 0,3844 & 0,6156 \\
\hline 18 & Mi vida tiene bastante interés & & 0,6477 & & 0,5197 & 0,4803 \\
\hline 14 & Tengo esperanza en el futuro & & 0,5357 & & 0,5782 & 0,4218 \\
\hline 16 & $\begin{array}{l}\text { Me resulta fácil tomar } \\
\text { decisiones }\end{array}$ & & 0,4781 & & 0,6399 & 0,3601 \\
\hline 20 & $\begin{array}{l}\text { Todavia disfruto con las } \\
\text { mismas cosas que antes } \\
\text { disfrutaba }\end{array}$ & & 0,4344 & & 0,5822 & 0,4178 \\
\hline 19 & $\begin{array}{l}\text { Siento que los demás estarian } \\
\text { mejor si yo muriera }\end{array}$ & & 0,4167 & & 0,6202 & 0,3798 \\
\hline 2 & $\begin{array}{l}\text { En la mañana es cuando me } \\
\text { siento mejor }\end{array}$ & & 0,4147 & & 0,7866 & 0,2134 \\
\hline 5 & Como igual que antes & & & 0,6381 & 0,5966 & 0,4034 \\
\hline 6 & $\begin{array}{l}\text { Mantengo mi deseo, interés } \\
\text { sexual y/o disfruto de las } \\
\text { relaciones sexuales }\end{array}$ & & & 0,6066 & 0,5447 & 0,4553 \\
\hline 11 & $\begin{array}{l}\text { Tengo la mente tan clara como } \\
\text { antes }\end{array}$ & & & 0,5608 & 0,6482 & 0,3518 \\
\hline 7 & Noto que estoy perdiendo peso & & & 0,5561 & 0,6636 & 0,3364 \\
\hline 12 & $\begin{array}{l}\text { Me resulta fácil hacer las cosas } \\
\text { que acostumbraba hacer }\end{array}$ & & & 0,4023 & 0,5801 & 0,4199 \\
\hline
\end{tabular}

Nota. ${ }^{*}$ Total de varianza explicada por el factor. Valores dentro de la tabla indican el nivel de correlación del componente con cada ítem. Componentes: componente 1 = afecto negativo, componente 2 = síntomas somáticos, componente 3 = síntomas cognitivos. Fuente: elaboración propia a partir del instrumento EZ-D publicado por Ruiz-Grosso et al. (13) 


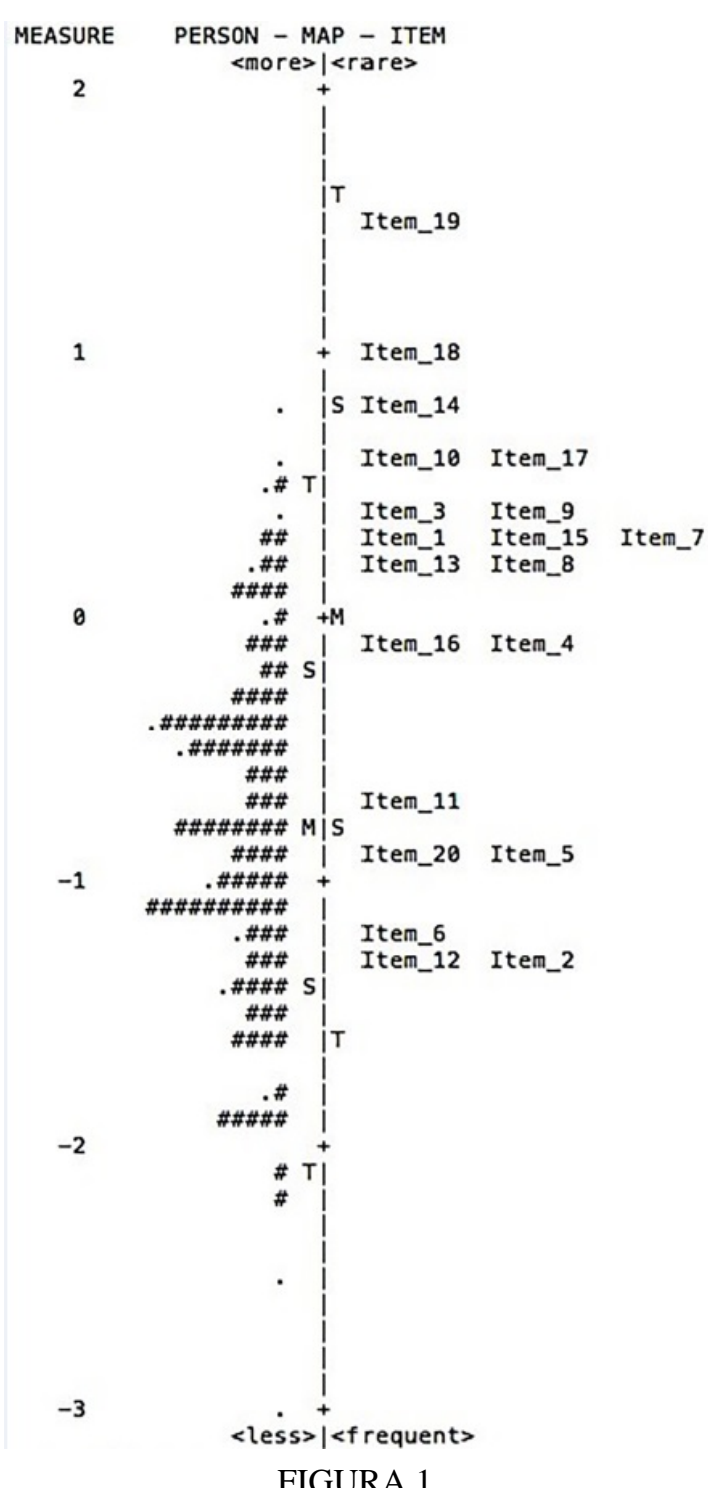

Mapa Wright escala de Zung para depresión (20 ítems) en pacientes con falla cardíaca ( $\mathrm{n}=200)$ Nota. Mapa del nivel de depresión de las personas y la capacidad de los ítems para medir depresión. Los valores varían entre $-4 a+2$ logits en la muestra evaluada. A la izquierda los numerales representan las personas del estudio ( $\mathrm{n}$ $=200)$. La ubicación del numeral o punto indica el nivel de depresión de las personas evaluadas. A la derecha se observan los 20 ítems de la versión de la escala original cuya posición representa su capacidad para medir depresión.

Fuente: elaboración propia

Con respecto a la EZ-D versión abreviada de veinte ítems, se identificaron trece $(1,3,4,10,11,12,13,14$, $16,17,18,19$ y 20) con coeficientes de correlación $\geq 0,50$ después de explorar la correlación lineal entre el puntaje total EZ-D y cada uno de los ítems (tabla 2). Esta nueva versión obtuvo una consistencia interna $\alpha$ $=0,819$ (IC $95 \% 0,783$ a 0,855). La prueba de esfericidad de Bartlett fue $\chi 2(78)=732,11, p=0,000$ y un KMO general de 0,807. Los ítems correlacionaron con un único factor que explicó el 33,54\% de la varianza total (tabla 3). El mapa de Wright confirma que los ítems 19 y 12 son el más difícil y fácil, respectivamente. 
TABLA 2.

Coeficientes de correlación de Pearson y Spearman del puntaje total de EZ-D con cada uno de los ítems $(\mathrm{n}=200)$

\begin{tabular}{llcc}
\hline & \multicolumn{1}{c}{ Ítems } & Pearson* & Spearman \\
\hline 1 & Me siento decaído y triste & $\mathbf{0 , 6 1 8 6}$ & $\mathbf{0 , 6 1 3 0}$ \\
\hline 2 & En la mañana es cuando me siento mejor & 0,0910 & 0,0379 \\
\hline 3 & Tengo periodos de llanto o deseos de llorar & $\mathbf{0 , 5 4 3 3}$ & $\mathbf{0 , 5 0 4 1}$ \\
\hline 4 & Me cuesta trabajo dormirme en la noche & $\mathbf{0 , 5 1 1 5}$ & $\mathbf{0 , 4 9 9 7}$ \\
\hline 5 & Como igual que antes & 0,3800 & 0,3890 \\
\hline 6 & $\begin{array}{l}\text { Mantengo mi deseo, interés sexual y/o disfruto de las } \\
\text { relaciones sexuales }\end{array}$ & 0,4688 & 0,4705 \\
\hline 7 & Noto que estoy perdiendo peso & 0,3485 & 0,3485 \\
\hline 8 & Tengo molestias de estreńimiento & 0,3326 & 0,3287 \\
\hline 9 & El corazón me late más rápido que de costumbre & 0,4101 & 0,3519 \\
\hline 10 & Me canso, aunque no haga nada & $\mathbf{0 , 6 1 1 1}$ & $\mathbf{0 , 6 1 1 9}$ \\
\hline 11 & Tengo la mente tan clara como antes & 0,4742 & $\mathbf{0 , 5 0 8 3}$ \\
\hline 12 & Me resulta fácil hacer las cosas que acostumbraba hacer & $\mathbf{0 , 6 1 4 7}$ & $\mathbf{0 , 6 4 1 2}$ \\
\hline 13 & Me siento intranquilo y no puedo mantenerme quieto & $\mathbf{0 , 5 6 6 5}$ & $\mathbf{0 , 5 7 7 1}$ \\
\hline 14 & Tengo esperanza en el futuro & $\mathbf{0 , 5 2 1 1}$ & $\mathbf{0 , 5 2 3 8}$ \\
\hline 15 & Estoy más irritable de lo usual & 0,4168 & 0,3387 \\
\hline 16 & Me resulta fácil tomar decisiones & $\mathbf{0 , 5 3 3 1}$ & $\mathbf{0 , 5 5 1 0}$ \\
\hline 17 & Siento que soy útil y necesario & $\mathbf{0 , 5 0 4 3}$ & $\mathbf{0 , 5 2 0 6}$ \\
\hline 18 & Mi vida tiene bastante interés & $\mathbf{0 , 4 9 7 7}$ & $\mathbf{0 , 5 0 2 4}$ \\
\hline 19 & Siento que los demás estarían mejor si yo muriera & $\mathbf{0 , 5 0 1 0}$ & 0,4430 \\
\hline 20 & Todavía disfruto con las mismas cosas que antes disfrutaba & $\mathbf{0 , 6 2 5 4}$ & $\mathbf{0 , 6 3 3 2}$ \\
\hline
\end{tabular}

Nota. ${ }^{*}$ Coeficiente de correlación. EZ-D = Escala de Zung para depresión. Correlaciones resaltadas alcanzaron valores $\geq 0,50$. Fuente: elaboración propia a partir del instrumento EZ-D publicado por Ruiz- Grosso et al. (13)

TABLA 3.

Análisis factorial de componentes principales con rotación oblicua de la escala de Zung para depresión (abreviada) en pacientes con falla cardíaca $(\mathrm{n}=200)$

\begin{tabular}{|c|c|c|c|c|}
\hline & \multirow{3}{*}{ Ítems } & \multirow{3}{*}{$\begin{array}{c}\text { Componente } \\
1 \\
33,54 \%^{*} \\
\end{array}$} & \multirow{3}{*}{ Singularidad } & \multirow{3}{*}{ Comunalidad* } \\
\hline & & & & \\
\hline & & & & \\
\hline 1 & Me siento decaído y triste & 0,6737 & 0,5462 & 0,4538 \\
\hline 3 & Tengo periodos de llanto o deseos de llorar & 0,5837 & 0,6592 & 0,3408 \\
\hline 4 & Me cuesta trabajo dormirme en la noche & 0,5134 & 0,7364 & 0,2636 \\
\hline 10 & Me canso, aunque no haga nada & 0,5996 & 0,6404 & 0,3596 \\
\hline 11 & Tengo la mente tan clara como antes & 0,4196 & 0,8239 & 0,1761 \\
\hline 12 & $\begin{array}{l}\text { Me resulta fácil hacer las cosas que } \\
\text { acostumbraba hacer }\end{array}$ & 0,5812 & 0,6622 & 0,3378 \\
\hline 13 & $\begin{array}{l}\text { Me siento intranquilo y no puedo } \\
\text { mantenerme quieto }\end{array}$ & 0,6031 & 0,6363 & 0,3637 \\
\hline 14 & Tengo esperanza en el futuro & 0,5982 & 0,6421 & 0,3579 \\
\hline 16 & Me resulta fácil tomar decisiones & 0,5482 & 0,6994 & 0,3006 \\
\hline 17 & Siento que soy útil y necesario & 0,5661 & 0,6795 & 0,3205 \\
\hline 18 & Mi vida tiene bastante interés & 0,5939 & 0,6473 & 0,3527 \\
\hline 19 & $\begin{array}{l}\text { Siento que los demás estarían mejor si yo } \\
\text { muriera }\end{array}$ & 0,5953 & 0,6456 & 0,3544 \\
\hline 20 & $\begin{array}{l}\text { Todavía disfruto con las mismas cosas que } \\
\text { antes disfrutaba }\end{array}$ & 0,6155 & 0,6211 & 0,3789 \\
\hline
\end{tabular}

Nota. *'Total de varianza explicada por el factor. Valores dentro de la tabla indican el nivel de correlación del componente con cada ítem.

Fuente: elaboración propia a partir del instrumento EZ-D publicado por Ruiz- Grosso et al. (13) 


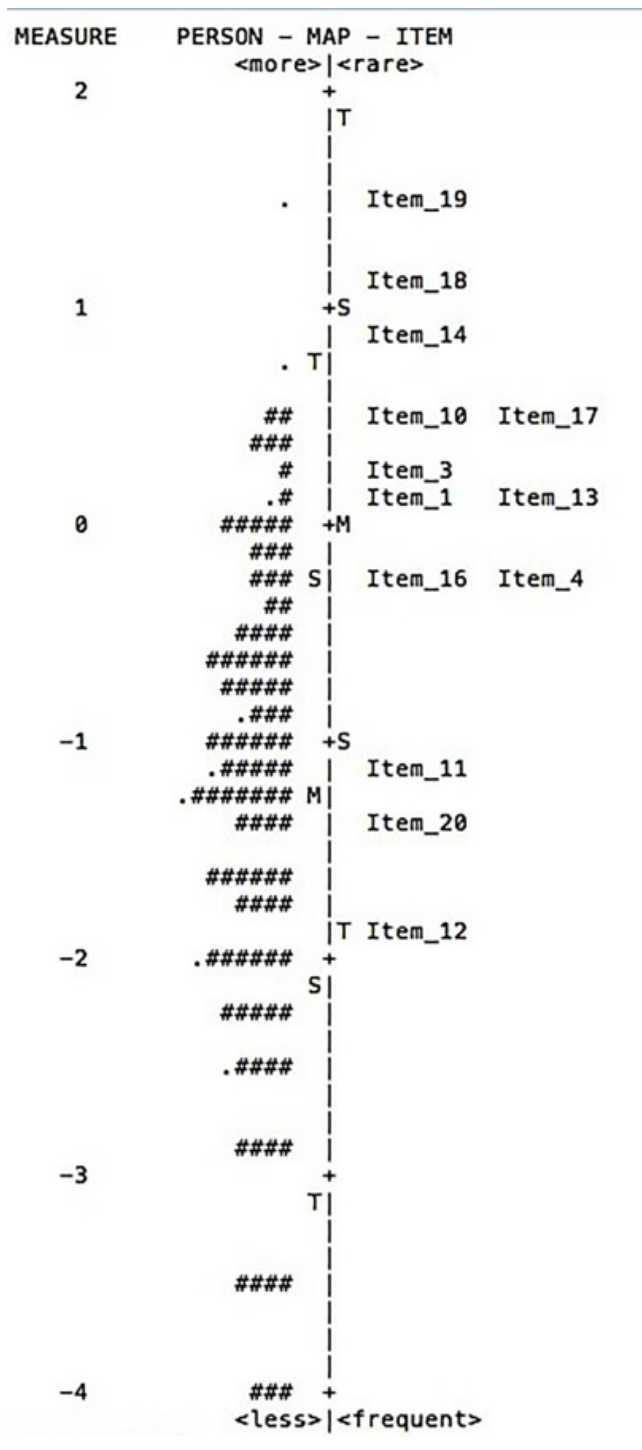

FIGURA 2.

Mapa Wright escala de Zung para depresión (13 ítems) en pacientes con falla cardíaca ( $\mathrm{n}=200)$ Nota. Mapa del nivel de depresión de las personas y la capacidad de los ítems para medir depresión. Los valores varían entre $-4 \mathrm{a}+2$ logits en la muestra evaluada. A la izquierda los numerales representan las personas del estudio $(\mathrm{n}=200)$. La ubicación del numeral o punto indica el nivel de depresión de las personas evaluadas. A la derecha se observan los 13 ítems de la versión abreviada cuya posición representa su capacidad para medir depresión.

Fuente: elaboración propia

\section{Discusión}

Hemos evaluado la validez de constructo de la EZ-D, versión original y abreviada, en pacientes ambulatorios con FC. Nuestros principales hallazgos son que tanto la EZ-D original como la abreviada tienen una buena consistencia interna. Además, en la versión original se identificaron tres componentes que explican el 51,59\% de la varianza total, mientras que en la abreviada se observó un único factor que explicó el 33,54\%. También logramos identificar que el ítem más difícil de responder fue "siento que los demás estarían mejor si yo muriera" y el más fácil fue "me resulta fácil hacer las cosas que acostumbraba a hacer".

Nuestros hallazgos son similares a los encontrados por Shafer (12), quien, en un metaanálisis que incluyó 13 estudios con un total de 12.621 participantes, de los cuales el $45 \%$ fueron realizados en pacientes, identificó 
tres factores con autovalores superiores a 1,4 que explicaban el $60 \%$ de la varianza total. Asimismo, en este estudio se puede evidenciar que la moda de los componentes entre los estudios metaanalizados fue de 3 factores, (rango 2-5) y el promedio del porcentaje de varianza explicada por estos factores fue del $43 \%$ (12).

Por otra parte, la consistencia interna aquí reportada fue similar a la de estudios realizados en población colombiana 0,85 (19) y 0,83 (21). Además, se destaca que el ítem 19, pese a los valores limítrofes de correlación con el puntaje total de la escala, se posicionó como el más difícil, tanto en la versión completa como en la abreviada, de igual forma el ítem 12 mantuvo su lugar como el ítem más fácil en ambas versiones.

Otro trabajo con resultados similares a los nuestros fue el realizado por Pérez et al. (36), quienes, tras aplicar la escala en una población mixta de personas sanas y con enfermedad coronaria, reportaron una solución con 3 factores tras aplicar una rotación Promax, con los cuales se coincidió en la distribución de 18 ítems. Solo se observó discordancia en los ítems 2 "en la mañana es cuando me siento mejor" y 7 "noto que estoy perdiendo peso", de igual forma se resalta que en dicho estudio, al realizar un análisis en el grupo de pacientes con enfermedad coronaria, la distribución factorial no se mantuvo.

El estudio de Campo et al. (22) es la investigación colombiana que tiene mayor similitud con nuestro estudio en cuanto a características sociodemográficas (edad, sexo, nivel de escolaridad, ocupación y estado civil), encontraron una consistencia interna de 0,832 e identificaron 2 factores que explicaban el $36 \%$ de la varianza. En un subanálisis según género, realizado por el mismo grupo investigador (37), se determinó una consistencia interna para mujeres de 0,816 y de 0,803 para hombres. El ACP estableció 2 factores para las mujeres (varianza 34,7 \%) y 3 factores para los hombres (varianza 46,8\%); resultados que guardan cierta consistencia con nuestros hallazgos.

De igual forma, Campo et al. (21), en la misma población general de Bucaramanga, diseñaron una EZ$\mathrm{D}$ abreviada donde identificaron 10 ítems con correlaciones superiores a 0,50 , que representaban un único factor y explicaban el $36,6 \%$ de la varianza, con una consistencia interna de 0,803 , la cual también conservó las propiedades psicométricas de la versión original, como en nuestro estudio. También es de resaltar que coincidimos en 8 de los ítems seleccionados para la versión abreviada y discrepamos en los siguientes 5: "me cuesta trabajo dormirme en la noche", "tengo esperanza en el futuro", "me resulta fácil tomar decisiones", "siento que soy útil y necesario" y "siento que los demás estarían mejor si yo muriera".

La asociación entre la FC y la depresión mayor se ha abordado desde múltiples perspectivas; desde el punto de vista fisiopatológico, los procesos relacionados con la disfunción cardíaca autónoma, el aumento de agentes proinflamatorios y moléculas de apoptosis soluble, así como la alteración de la quimiotaxis y el incremento de la actividad neuroendocrina, entre otros, contribuyen al desarrollo de esta condición $(38,39)$. Adicionalmente, es importante mencionar que existe un círculo vicioso entre la FC y el trastorno depresivo debido a que este último, con su impacto fisiológico y conductual, tiende a empeorar la FC en términos de sus resultados clínicos. A su vez, la FC, como condición crónica con un curso prolongado, presencia de múltiples síntomas físicos no deseados y un impacto negativo en la calidad de vida, conduce, con frecuencia, al desarrollo de cuadros depresivos (40).

Para finalizar, existen varias fortalezas y limitaciones de este estudio. En nuestro conocimiento, es la primera vez que se revisa la validez de constructo del EZ-D y se propone una versión corta en pacientes ambulatorios con FC. Adicionalmente, destacamos el adecuado tamaño de muestra y la buena consistencia interna del instrumento, demostrada en el valor global del estadístico KMO y el alfa de Cronbach, respectivamente, lo que garantizó la estabilidad de las medidas obtenidas. Además, identificamos los ítems del EZ-D más difíciles y fáciles de responder. Sin embargo, existen algunas limitaciones importantes a considerar. En primer lugar, el estudio fue realizado en un solo centro de FC, por lo tanto, los resultados no pueden ser extrapolados a todas las clínicas de FC de Colombia. En segundo lugar, la escala de EZ-D es un cuestionario autoadministrado, y en nuestro estudio fue aplicado por una enfermera, debido a que un alto porcentaje de participantes tenían un bajo nivel educativo, no obstante, creemos que esto no afectó la medición del estado depresivo evidenciado 
en una buena consistencia interna tanto en la versión original como en la versión corta. Finalmente, se trató de una muestra no probabilística, que puede tener implicaciones en la representatividad de la población.

La validación de constructo de la ED-Z permite responder a las necesidades de la práctica clínica de enfermería de tener instrumentos que faciliten la evaluación y seguimiento de la depresión en los pacientes con FC, además, el uso de un instrumento validado permite la implementación de intervenciones de enfermería oportunas y eficientes, lo cual tiene un impacto positivo, teniendo en cuenta que la depresión se ha asociado con desenlaces de importancia clínica en esta población.

\section{Conclusiones}

En conclusión, se observó una buena validez de constructo tanto para la EZ-D, en su versión original, como para la versión abreviada creada en la población estudiada. Sin embargo, se requieren estudios adicionales que verifiquen estos hallazgos en una muestra representativa (probabilística y multicéntrica), y que otros aspectos de la psicometría, como la validez de criterio, la reproducibilidad y la sensibilidad al cambio, sean evaluados.

\section{Referencias}

1. Ishak WW, Edwards G, Herrera N, Lin T, Hren K, Peterson M, et al. Depression in Heart Failure: A Systematic Review. Innov Clin Neurosci [Internet]. 2020;17(4-6):27-38. Disponible en: https://www.ncbi.nlm.nih.gov/p $\mathrm{mc} /$ articles/PMC7413333/

2. Jia Z, Du X, Du J, Xia S, Guo L, Su X, et al. Prevalence and factors associated with depressive and anxiety symptoms in a Chinese population with and without cardiovascular diseases. J Affect Disord [Internet]. 2021;286:241-247. https://doi.org/10.1016/j.jad.2021.02.006

3. Celano CM, Villegas AC, Albanese AM, Gaggin HK, Huffman JC. Depression and Anxiety in Heart Failure: A Review. Harv Rev Psychiatry [Internet]. 2018;26(4):175-184. http://doi.org/10.1097/HRP.00000000000001 62

4. Freedland KE, Carney RM, Rich MW, Steinmeyer BC, Rubin EH. Cognitive behavior therapy for depression and self-care in heart failure patients a randomized clinical trial. JAMA Intern Med [Internet]. 2015;175(11):1773-1782. http://doi.org/10.1001/jamainternmed.2015.5220

5. Rutledge T, Reis VA, Linke SE, Greenberg BH, Mills PJ. Depression in heart failure a meta-analytic review of prevalence, intervention effects, and associations with clinical outcomes. J Am Coll Cardiol [Internet]. 2006;48(8):1527-1537. http://doi.org/10.1016/j.jacc.2006.06.055

6. Mbakwem AC, Aina OF. Comparative study of depression in hospitalized and stable heart failure patients in an urban Nigerian teaching hospital. Gen Hosp Psychiatry [Internet]. 2008;30(5):435-440. https://doi.org/10.10 16/j.genhosppsych.2008.04.008

7. Newhouse A, Jiang W. Heart failure and depression. Heart Fail Clin [Internet]. 2014;10(2):295-304. https://doi. org/10.1016/j.hfc.2013.10.004

8. Yancy CW, Jessup M, Bozkurt B, Butler J, Casey DE Jr., Drazner MH, et al. 2013 ACCF/AHA guideline for the management of heart failure: a report of the American College of Cardiology Foundation/American Heart Association Task Force on Practice Guidelines. J Am Coll Cardiol [Internet]. 2013;62(16):e147-e239. https:/ /doi.org/10.1016/j.jacc.2013.05.019

9. Polikandrioti M, Koutelekos I, Panoutsopoulos G, Gerogianni G, Zartaloudi A, Dousis E, et al. Hospitalized patients with heart failure: the impact of anxiety, fatigue, and therapy adherence on quality of life. Arch Med Sci Atheroscler Dis [Internet]. 2019;4:e268-e279. https://doi.org/10.5114/amsad.2019.90257

10. Albert NM, Fonarow GC, Abraham WT, Gheorghiade M, Greenberg BH, Nunez E, et al. Depression and clinical outcomes in heart failure: an OPTIMIZE-HF analysis. Am J Med [Internet]. 2009;122(4):366-373. https://d oi.org/10.1016/j.amjmed.2008.09.046 
11. Martensson J, Stromberg A, Dahlstrom U, Karlsson JE, Fridlund B. Patients with heart failure in primary health care: effects of a nurse-led intervention on health-related quality of life and depression. Eur J Heart Fail [Internet]. 2005;7(3):393-403. https://doi.org/10.1016/j.ejheart.2004.01.016

12. Shafer AB. Meta-analysis of the Factor Structures of Four Depression Questionnaires. J Clin Psychol [Internet]. 2006;62(1):123-146. https://doi.org/10.1002/jclp.20213

13. Ruiz-Grosso P, Mola CLD, Vega-Dienstmaier JM, Arévalo JM. Validation of the Spanish Center for Epidemiological Studies Depression and Zung Self-Rating Depression Scales: A Comparative Validation Study. PLoS ONE [Internet]. 2012;7(10):e45413. https://doi.org/10.1371/journal.pone.0045413

14. Kourkoveli P, Rammos S, Parissis J. Depressive Symptoms in Patients with Congenital Heart Disease: Incidence and Prognostic Value of Self-Rating Depression Scales. Congenit Heart Dis [Internet]. 2015;10(3):240-247. h ttps://doi.org/10.1111/chd.12200

15. Parissis J, Karavidas A, Farmakis D, Papoutsidakis N, Matzaraki V, Arapi S, et al. Efficacy and safety of functional electrical stimulation of lower limb muscles in elderly patients with chronic heart failure: A pilot study. Eur J Prev Cardiol [Internet]. 2015;22(7):831-836. https://doi.org/10.1177/2047487314540546

16. Suzuki T, Shiga T, Kuwahara K. Impact of clustered depression and anxiety on mortality and rehospitalization in patients with heart failure. J Cardiol [Internet]. 2014;64(6):456-462. https://doi.org/10.1016/j.jjcc.2014.02.0 31

17. Cogollo Z, Díaz CE, Campo A. Exploración de la validez de constructo de la escala de Zung para depresión en adolescentes escolarizados. Colomb Med (Cali). 2006;37(2):102-106.

18. Lezama-Meneses SR. Propiedades psicométricas de la escala de Zung para síntomas depresivos en población adolescente escolarizada colombiana. Psychol. 2012;6(1):91-101.

19. Campo-Arias A, Díaz-Martínez LA, Rueda-Jaimes GE, Barros-Bermúdez JA. Validación de la escala de Zung para depresión en universitarias de Bucaramanga, Colombia. Rev Colomb Psiquiatr. 2005;34(1):54-62.

20. Díaz LA, Campo A, Rueda GE, Barros JA. Propuesta de una versión abreviada de la escala de Zung para depresión. Colmb Med (Cali). 2005;36(3):168-172.

21. Campo A, Díaz LA, Rueda GE. Validez de la escala breve de Zung para tamizaje del episodio depresivo mayor en la población general de Bucaramanga, Colombia. Biomédica [Internet]. 2006;26:415-423. https://doi.org/10. 7705/biomedica.v26i3.360

22. Campo-Arias A, Díaz-Martínez L, Rueda-Jaimes G, Cadena L, Hernández N. Validation of Zung's self-rating depression scale among the Colombian general population. Soc Behav Pers [Internet]. 2006;34(1):87-94. http s://doi.org/10.2224/sbp.2006.34.1.87

23. Jokelainen J, Timonen M, Keinanen-Kiukaanniemi S, Harkonen P, Jurvelin H, Suija K. Validation of the Zung selfrating depression scale (SDS) in older adults. Scand J Prim Health Care [Internet]. 2019;37(3):353-357. https ://doi.org/10.1080/02813432.2019.1639923

24. Zung WWK. A Self-Rating Depression. Arch Gen Psychiatry [Internet]. 1965;12:63-70. https://doi.org/10.100 1/archpsyc.1965.01720310065008

25. Zung WWK. From Art to Science Seeet. Arch Gen Psychiatry [Internet]. 1973;29(3):328-337. https://doi.org/ 10.1001/archpsyc.1973.04200030026004

26. Romera I, Delgado-Cohen H, Pérez T, Caballero L, Gilaberte I. Factor analysis of the Zung self-rating depression scale in a large sample of patients with major depressive disorder in primary care. BMC Psychiatry [Internet]. 2008;8(4):1-8. https://doi.org/10.1186/1471-244X-8-4

27. Achury-Saldaña D, Garavito-Amaya C, Gómez-Rodríguez J, Muñoz-Bolaños S. Cuidado de enfermería al paciente con falla cardiaca en situación de depresión. Investig Enferm Imagen Desarr. 2011;13(1):47-64.

28. Cronbach JL. Coefficient alpha and the internal structre of test. Psychometrika. 1951;16(3):297-298.

29. Souza AC, Alexandre NMC, Guirardello EB. Psychometric properties in instruments evaluation of reliability and validity. Epidemiol Serv Saude [Internet]. 2017;26(3):649-659. https://doi.org/10.5123/S1679-49742017000 300022 
30. Kaiser HF. An Index of Factorial Simplicity. Phsychometrika. 1974;39(1):31-32.

31. Bartlett MS. Tests of Significance in Factor Analysis. Br. J. Stat. Psychol. 1950;3(2):77-85.

32. Lloret-Segura S, Ferreres-Traver A, Hernández-Baeza A, Tomás-Marco I. El análisis factorial exploratorio de los ítems: una guía práctica, revisada y actualizada. An. de Psicol. 2014;30(3):1151-1169.

33. Gorsuch RL. Exploratory factor analysis: its role in item analysis. J Pers Assess [Internet]. 1997;68(3):532-560. h ttps://doi.org/10.1207/s15327752jpa6803_5

34. Van der Wal M, Tuinebreijer W, Bloemen M, Verhaegen P, Middelkoop E, van Zuijlen P. Rasch analysis of the Patient and Observer Scar Assessment Scale (POSAS) in burn scars. Qual Life Res [Internet]. 2012;21:13-23. h ttps://doi.org/10.1007/s11136-011-9924-5

35. Rojas-Sánchez LZ, Hernández-Vargas JA, Trujillo-Cáceres SJ, Roa-Díaz ZM, Jurado-Arenales AM, Toloza-Pérez YG. Usefulness of the Diagnosis Decreased Cardiac Output (00029); in Patients With Chronic Heart Failure. Int J Nurs Knowl [Internet]. 2017;28(4):192-198. https://doi.org/10.1111/2047-3095.12148

36. Pérez-Adel P, Fernández, II, Ojeda FB, González RD, Gaos Meizoso MT. Zung scale factor invariance in male coronary patients and healthy people. Span J Psychol [Internet]. 2013;16:E72. https://doi.org/10.1017/sjp.20 13.62

37. Díaz-Martínez L, Barros-Bermúdez J, Cadena L, Rueda-Jaimes G, Hernández N, Campo-Arias A. Factor analysis of the Zung' self-rating depression scale among the general population from Bucaramanga, Colombia: a gender comparison. Arch Health. 2008;3:7-12.

38. Michalakeas CA, Parissis JT, Douzenis A, Nikolaou M, Varounis C, Andreadou I, et al. Effects of sertraline on circulating markers of oxidative stress in depressed patients with chronic heart failure: a pilot study. J Card Fail [Internet]. 2011;17(9):748-754. https://doi.org/10.1016/j.cardfail.2011.05.004

39. Corbineau S, Breton M, Mialet-Pérez J, Costemale-Lacoste JF. Major depression and heart failure: Interest of monoamine oxidase inhibitors. Int J Cardiol [Internet]. 2017;247:1-6. https://doi.org/10.1016/j.ijcard.2017.0 7.005

40. Ghosh RK, Ball S, Prasad V, Gupta A. Depression in heart failure: Intricate relationship, pathophysiology and most updated evidence of interventions from recent clinical studies. Int J Cardiol [Internet]. 2016;224:170-177. htt ps://doi.org/10.1016/j.ijcard.2016.09.063

\section{Notas}

* Artículo original de investigación

\section{Licencia Creative Commons CC BY 4.0}

Cómo citar este artículo: Rojas LZ, Trujillo-Cáceres SJ, Hernández-Vargas JA, Jurado AM, Echeverría LE, Gómez-Ochoa SA, Roa-Díaz ZM. Validación de constructo de la escala Zung en pacientes con falla cardíaca. Investig Enferm Imagen Desarr. 2021;23. https://doi.org/10.11144/Javeriana.ie23.vcez 